\title{
COVID-19: Manifestaciones clínicas y diagnóstico
}

\author{
COVID-19: Clinical features and diagnosis \\ Idalia Parra-Avila* \\ * Departamento de Trasplantes. Unidad Médica de Alta Especialidad, IMSS Mérida, Yucatán.
}

\section{RESUMEN}

En población general el cuadro clínico de la infección por SARSCoV-2 se caracteriza por síntomas como fiebre, tos y fatiga, el tiempo de incubación en promedio va de cinco a seis días hasta 14 días, generalmente son casos leves que ameritan manejo ambulatorio y sólo $30 \%$ requerirá tratamiento intrahospitalario. Los principales factores de riesgo para enfermedad severa descritos son: edad $\geq 65$ años, obesidad, hipertensión, diabetes mellitus, enfermedad coronaria, enfermedad pulmonar obstructiva y neoplasias. Se ha reportado una mortalidad general alrededor de $5 \%$, pero puede ser mayor en casos de pacientes críticamente enfermos. En el escenario de receptores de trasplante, al estar bajo inmunosupresión, se espera que el cuadro clínico y hallazgos de laboratorio e imagen suelan ser distintos a lo encontrado en población general, como se ha observado con otro tipo de infecciones. Sin embargo, en varios de los reportes hasta ahora publicados, en general, no hay diferencias con población no trasplantada. A pesar de que el cuadro clínico pueda ser leve, la frecuencia de complicaciones es más común en población trasplantada comparada con población general. La mortalidad reportada alcanza el $20 \%$, lo cual es mayor que en la población general. El diagnóstico molecular con reacción en cadena de la polimerasa con transcriptasa inversa (RT-PCR) se considera el estándar de oro; no obstante, su sensibilidad está por debajo del $80 \%$. Sin duda, las circunstancias epidemiológicas, la sospecha clínica, RT-PCR y los estudios de imagen son complementarios a la hora de establecer un diagnóstico correcto.

Palabras clave: COVID-19, manifestaciones clínicas, cuadro clínico, diagnóstico, RT-PCR.

\section{MANIFESTACIONES CLÍNICAS DE COVID-19}

En población general el cuadro clínico más frecuente se caracteriza por síntomas leves como fiebre, tos y fatiga, el tiempo de incubación en promedio va de cinco a seis días hasta 14 días. Generalmente corresponden

\begin{abstract}
In general population, the clinical picture of SARS-CoV-2 infection is characterized by symptoms such as fever, cough and fatigue. Incubation period ranges from 5 or 6 days to 14 days, generally they are mild cases with outpatient management and only $30 \%$ will be admitted in hospital. Main risk factors for severe disease are: age $\geq 65$ years, obesity, arterial hypertension, diabetes mellitus, coronary heart disease, obstructive pulmonary disease, and malignancies. General mortality is around 5\% but may be higher in critically ill patients. In transplant recipients, who are under immunosuppression, it is expected that clinical picture, laboratory and imaging findings could be different compared with general population, as has been observed with other kind of infections. However, in several published reports, there are no differences with non-transplanted population. Although clinical picture may be mild, frequency of complications is more common in transplanted population. Mortality is around $20 \%$, higher than non-transplanted population. Molecular diagnosis with RT-PCR is defined as the gold standard, however its sensitivity is below 80\%. Undoubtedly, the epidemiological circumstances, clinical suspicion, $R T-P C R$ report and imaging tests are complementary to establishing a correct diagnosis.
\end{abstract}

Keywords: COVID-19, clinical manifestations, clinical picture, diagnosis, RT-PCR.

a casos leves manejados con cuidados ambulatorios y sólo 30\% requerirá tratamiento intrahospitalario por su presentación como neumonía atípica y que puede complicarse con síndrome de distrés respiratorio agudo secundario al efecto citopático del virus en el tracto respiratorio y la respuesta inmune del huésped con la 
liberación de una tormenta de citocinas; asimismo, se espera una mortalidad general alrededor de $5 \%$, pero puede ser mayor en casos de pacientes críticamente enfermos. Los principales factores de riesgo para enfermedad severa descritos son: edad $\geq 65$ años, hipertensión, diabetes mellitus, enfermedad coronaria, enfermedad pulmonar obstructiva y neoplasias. ${ }^{1-4}$

Dentro de los pocos reportes locales hasta ahora publicados, Bello-Chavolla ${ }^{5}$ y colegas describen en un registro de 15,529 casos confirmados un predominio del sexo masculino (58\%) acorde a otros reportes, sin embargo, con una presentación a edad más joven (46.5 \pm 15.1$)$, con predominio de comorbilidades como diabetes mellitus, obesidad e hipertensión, en el que sólo una tercera parte fueron casos leves y menos de $5 \%$ requirió apoyo de cuidados intensivos, con una mortalidad general alrededor de $9 \%$, un poco más alta comparada a la encontrada en otros reportes, tal como se describe en la Tabla 1.

En el escenario de los receptores de trasplante, al estar bajo inmunosupresión, se espera que el cuadro clínico y hallazgos de laboratorio e imagen suelan ser distintos a lo encontrado en población general, como se ha observado con otro tipo de infecciones. Sin embargo, en varios de los reportes hasta ahora publicados, no hay diferencias con población no trasplantada. Incluso se ha

Tabla 1: Características demográficas, clínicas y desenlaces en población general con infección por SARS-CoV-2.

\begin{tabular}{|c|c|c|c|c|c|c|c|c|}
\hline \multicolumn{9}{|c|}{ Población general } \\
\hline & \multicolumn{2}{|c|}{ China } & \multicolumn{2}{|c|}{ Europa } & \multicolumn{2}{|c|}{ EUA } & \multicolumn{2}{|c|}{ México } \\
\hline & $\begin{array}{c}\text { Guan W, } \\
\text { et al } \\
(n=1,099)\end{array}$ & $\begin{array}{c}\text { WuZ, } \\
\text { et al'0 } \\
(\mathrm{n}=72,314)\end{array}$ & $\begin{array}{c}\text { Lechien JR, } \\
\quad \text { et a l|1 } \\
(n=1,420)\end{array}$ & $\begin{array}{c}\text { Grasselli G, } \\
\text { et a al'12 } \\
(n=1,591)\end{array}$ & $\begin{array}{l}\text { Bialek S, } \\
\text { et a } \text { al'3 }^{13} \\
(n=4,226)\end{array}$ & $\begin{array}{l}\text { Arentz M, } \\
\text { et a }\left.\right|^{14} \\
(n=21)\end{array}$ & $\begin{array}{c}\text { Bello-Chavolla } \\
\text { OY, et al } \\
(n=15,529)\end{array}$ & $\begin{array}{l}\text { Mejia-Vilet } \\
\text { JM, et a }\left.\right|^{15} \\
(n=329)\end{array}$ \\
\hline Hombres (\%) & 58.0 & $\mathrm{~N} / \mathrm{D}$ & 32.3 & 82 & N/D & 52.0 & 57.8 & 64 \\
\hline Edad, años & $47(35-58)$ & $30-79(87 \%)$ & $39.1 \pm 12.09$ & $63(56-70)$ & $31 \%(>65)$ & $70(43-92)$ & $46.5 \pm 15.1$ & $49(41-60)$ \\
\hline Comorbilidades (\%) & 23.7 & $N / D$ & & 68 & N/D & 86.0 & & 70 \\
\hline DM & 7.4 & & 1.7 & 17 & & 33.3 & 18.2 & 24 \\
\hline HAS & 15.0 & & 9.2 & 49 & & & 21.7 & 27 \\
\hline ECV & 2.5 & & & 21 & & 42.9 & & \\
\hline ERC & 0.7 & & 0.4 & 3 & & 47.6 & & 6 \\
\hline EPOC & 1.1 & & & 4 & & 33.3 & 2.5 & \\
\hline Obesidad & $N / D$ & & & $N / D$ & & & 20.7 & 40 \\
\hline Tabaquismo & 12.6 & & 14.2 & $N / D$ & & & & 7 \\
\hline Sintomas (\%) & & $N / D$ & & $N / D$ & N/D & & N/D & \\
\hline Fiebre & 88.7 & & 45.4 & & & 52.0 & & 92 \\
\hline Tos & 67.8 & & 63.2 & & & 48.0 & & 87 \\
\hline Cefalea & 13.6 & & 70.3 & & & & & 49 \\
\hline Disnea & 18.7 & & 28.4 & & & 76.0 & & 70 \\
\hline Odinofagia & 13.9 & & 52.9 & & & & & 30 \\
\hline Obstrucción nasal & 4.8 & & 67.8 & & & & & $N / D$ \\
\hline Astenia/fatiga & 38.1 & & 63.3 & & & & & $N / D$ \\
\hline Mialgias & 14.9 & & 62.5 & & & & & 45 \\
\hline Diarrea & 3.8 & & 38.1 & & & & & 17 \\
\hline Linfopenia & 83.2 & $N / D$ & $N / D$ & $N / D$ & $N / D$ & 67.0 & & $N / D$ \\
\hline Falla renal & 0.5 & $\mathrm{~N} / \mathrm{D}$ & $N / D$ & $N / D$ & $\mathrm{~N} / \mathrm{D}$ & 19.1 & & $N / D$ \\
\hline Casos leves (\%) & 84.0 & 81.0 & $N / D$ & 20 & $N / D$ & 14.2 & 29.5 & \\
\hline Hospitalización (\%) & 100.0 & 14.0 & 8.1 & 100 & 12.0 & 19.0 & 38.9 & 65 \\
\hline UCl admisión (\%) & 5.0 & 5.0 & N/D & 100 & 2.8 & 81.0 & 4.4 & 35 \\
\hline VMA (\%) & $1.4-2.3$ & $N / D$ & $N / D$ & 88 & N/D & 71.0 & 4.3 & \\
\hline Mortalidad (\%) & 1.4 & 2.3 & $\mathrm{~N} / \mathrm{D}$ & & $1.8-3.4$ & 52.4 & 9.2 & \\
\hline Mortalidad en UCI (\%) & & 49.0 & & 26 & N/D & & & 53 \\
\hline
\end{tabular}

EUA = Estados Unidos de América; $N / D$ = no data; $D M$ = diabetes mellitus; $H A S$ = hipertensión arterial sistémica; $E C V$ = enfermedad cardiovascular; $E R C=$ enfermedad renal crónica; $E P O C$ = enfermedad pulmonar obstructiva crónica; Casos leves = no neumonía o neumonía leve; $\mathrm{UCl}=$ Unidad de Cuidados Intensivos; $\mathrm{VMA}$ = ventilación mecánica asistida. 
Tabla 2: Características demográficas, clínicas y desenlaces en receptores de trasplante de órgano sólido con infección por SARS-CoV-2.

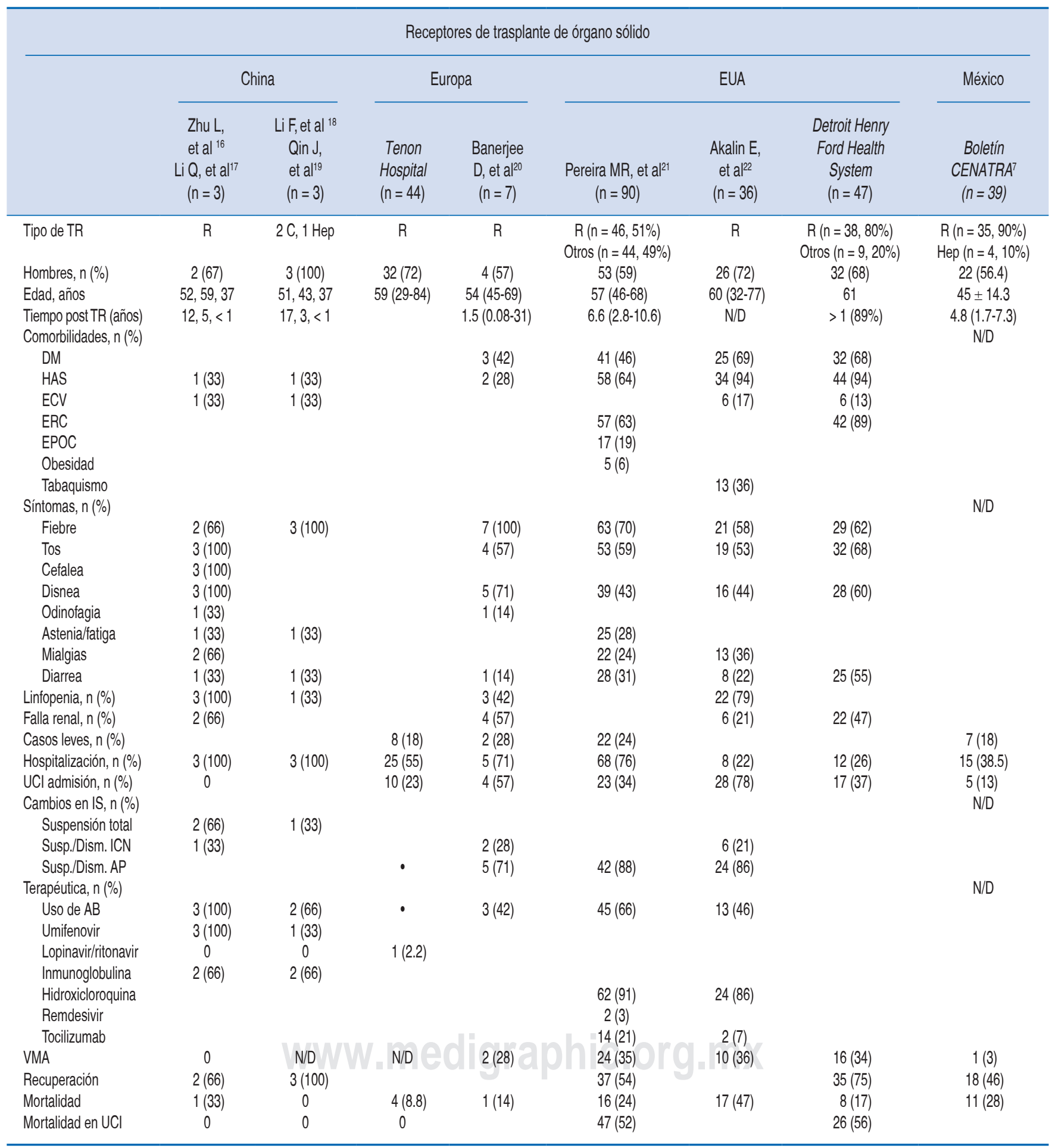

TR = trasplante; $\mathrm{R}$ = riñón; $\mathrm{C}$ = corazón; Hep = hígado; UCI = Unidad de Cuidados Intensivos; $\mathrm{N} / \mathrm{D}=$ no data; $(\bullet)$ = reportado sin frecuencia; $\mathrm{IS}$ = inmunosupresión; Susp. = suspensión; Dism. = disminución; $\mathrm{AP}=$ antiproliferativo; $\mathrm{ICN}$ = inhibidor de calcineurina; $\mathrm{AB}$ = antibiótico; $\mathrm{VMA}$ = ventilación mecánica asistida. 
postulado la hipótesis de que el paciente inmunocomprometido puede cursar con un cuadro menos agresivo por la interacción de los inmunosupresores con el bloqueo de la producción de citocinas. ${ }^{5}$ Por otro lado, la presencia de comorbilidades tales como diabetes mellitus, hipertensión o edad avanzada son factores de riesgo relacionados con un cuadro clínico agresivo en receptores de trasplante, similar a lo que sucede con la población general. ${ }^{3}$ A pesar de que el cuadro clínico pueda ser leve, la frecuencia de complicaciones es más común en población trasplantada comparada con población general.,7

En las series de casos hasta ahora reportadas, la edad de los receptores de trasplante fue de 37 a 77 años, la temporalidad de tiempo postrasplante incluyó desde 19 días a 31 años, los síntomas más comunes fueron fiebre, tos y disnea, una tercera parte presentó diarrea y entre $20-60 \%$ tuvo falla renal como complicación. Dos terceras partes ameritaron hospitalización, la mayoría requirió ajustes en la inmunosupresión, principalmente a expensas de la disminución o suspensión del antiproliferativo. El uso de inmunomoduladores como antivirales o hidroxicloroquina fue variable y acorde al protocolo de cada centro de trasplantes. La mortalidad fue $20 \%$ mayor comparado con población general. Datos locales obtenidos por el Centro Nacional de Trasplantes ${ }^{7}$ reportan 39 casos de receptores de trasplante (35 renal y 4 hepático), de los cuales $72 \%$ fueron casos confirmados, $8 \%$ casos sospechosos y $20 \%$ casos negativos, con edad promedio $45 \pm 14$ años, $56 \%$ fueron hombres, con una mediana de años postrasplante de 4.8 (1.7-7.3), 18\% recibió manejo ambulatorio, $38.5 \%$ requirió hospitalización y $13 \%$ ingresó a la UCl. Las entidades con mayor núme-

Tabla 3: Resultados obtenidos en los especímenes clínicos analizados por RT-PCR para la detección de SARS-CoV-2.

\begin{tabular}{lrccc}
\hline \multicolumn{1}{c}{ Sitio de muestreo } & $n$ & $\begin{array}{r}\text { Resultado } \\
\text { positivon (\%) }\end{array}$ & País & Referencia \\
\hline BAL & 15 & $14(93)$ & China & 8 \\
Biopsia por & 13 & $6(46)$ & & \\
cepillado bronquial & 104 & $75(72)$ & & \\
Esputo & 8 & $5(63)$ & & \\
Hisopado nasal & 398 & $126(32)$ & & \\
Hisopado faríngeo & 153 & $44(29)$ & & \\
Heces & 307 & $3(1)$ & & \\
Sangre & 72 & 0 & & \\
Orina & 6 & $3(60)$ & Francia & 9 \\
Hisopado nasofaríngeo & 6 & 0 & & \\
BAL & & & & \\
\hline
\end{tabular}

$\mathrm{BAL}=$ lavado bronquioalveolar.
Tabla 4: Interpretación de ensayo molecular y serológico.

\begin{tabular}{cccl}
\hline & \multicolumn{3}{c}{ Serológico } \\
\hline $\lg \mathrm{M}$ & $\lg \mathrm{M}$ & Molecular & \multicolumn{1}{c}{ Interpretación } \\
\hline+ & + & + & Infección en fase activa \\
+ & - & + & Infección temprana \\
- & - & + & Infección en periodo de ventana \\
- & + & + & Infección recurrente o tardía \\
+ & + & - & Infección en etapa de recuperación \\
- & + & - & Antecedente de infección \\
+ & - & - & Falso negativo (PCR), infección temprana \\
\hline
\end{tabular}

ro de casos fueron: Ciudad de México 24 casos (62\%), Coahuila 5 casos (13\%) y Puebla 4 casos (10.3\%). Las instituciones que brindaron atención a los casos principalmente fueron: Instituto Mexicano del Seguro Social 20 casos (51\%), Secretaría de Salud 13 casos (33\%) y hospitales privados 5 casos (13\%). En cuanto a los desenlaces, $46 \%$ se recuperó y la mortalidad fue de $28 \%$, tal como se muestra en la Tabla 2.

\section{DESEMPEÑO DE LAS PRUEBAS DIAGNÓSTICAS}

\section{RT-PCR}

La reacción en cadena de la polimerasa con transcriptasa inversa (RT-PCR, por sus siglas en inglés) por ahora es la prueba considerada como el estándar de oro para la detección de infección por SARS-CoV-2, debido a que una prueba positiva en un paciente enfermo tendrá una razón de verosimilitud muy alta; sin embargo, su sensibilidad es limitada, de accesibilidad variable y difiere entre cada país, hospital y laboratorio comercial.

Las principales limitaciones descritas recaen en la variabilidad del hisopado, medios de transporte y disponibilidad del ensayo. La frecuencia de falsos negativos va de 2 a $22 \%$, esto dependerá de variables como: muestra insuficiente, temporalidad del muestreo en una etapa muy temprana o tardía de la enfermedad, error de laboratorio o kit, sitio de muestreo (por ejemplo: nasal, oral, lavado bronquioalveolar [BAL], entre otros). ${ }^{8}$

En un estudio reciente por Wang y colaboradores, ${ }^{9}$ en 205 pacientes diagnosticados con COVID-19 fueron analizadas 1,070 muestras obtenidas de sangre, esputo, heces, orina y secreciones nasales, los resultados se describen en la Tabla 3.

Se identificó que las muestras obtenidas por BAL fueron las que tuvieron un alto porcentaje de prue- 
bas positivas (93\%), seguido de muestras en esputo $(72 \%)$, en cambio sólo fueron positivas 63 y $32 \%$ aquellas muestras obtenidas por hisopado nasal y faríngeo, respectivamente. Así pues, se concluye que no se cuenta con una prueba suficiente para excluir la infección por SARS-CoV-2 en muestras obtenidas por hisopado simultáneo nasal y faríngeo cuya sensibilidad dependerá del tipo de prueba y método de recolección de la muestra.

Por otro lado, lograron aislar ARN de SARS-CoV-2 en heces, sin embargo, esto no es suficiente para afirmar que sea una vía de transmisión, sobre todo porque los pacientes carecían de síntomas gastrointestinales o diarrea. Además, aunque fue poco frecuente, la po-

Tabla 5: Escala ABC-GOALS, sistema de puntaje.

\begin{tabular}{|c|c|c|c|}
\hline \multirow[b]{2}{*}{ Variables } & \multicolumn{3}{|c|}{ Puntaje } \\
\hline & Clínicas & $\begin{array}{l}\text { Clínicas y de } \\
\text { laboratorio }\end{array}$ & $\begin{array}{c}\text { Diagnóstico por } \\
\text { imagen }\end{array}$ \\
\hline Sexo Masculino & 1 & 1 & 1 \\
\hline A presión arterial sistólica & & & \\
\hline$<100 \mathrm{mmHg}$ & 4 & 4 & 4 \\
\hline $\begin{array}{l}\text { B Disnea } \\
\text { C Índice de Charlson }\end{array}$ & 1 & 1 & - \\
\hline 1-2 puntos & 1 & - & - \\
\hline$\geq 3$ puntos & 3 & 2 & 2 \\
\hline G Glucosa > $200 \mathrm{mg} / \mathrm{dL}$ & - & 2 & 2 \\
\hline $\begin{array}{l}0 \text { Obesidad, IMC > } 30 \\
\mathrm{~kg} / \mathrm{m}^{2}\end{array}$ & 2 & 2 & 2 \\
\hline A Albúmina $<3.5 \mathrm{~g} / \mathrm{dL}$ & - & 1 & - \\
\hline L Lactato deshidrogenasa* & - & 2 & 2 \\
\hline $\begin{array}{l}\mathrm{S} \mathrm{SaO}_{2} / \mathrm{FiO}_{2}<300 \\
\text { Afección pulmonar por }\end{array}$ & - & 4 & 3 \\
\hline $\mathrm{TAC}>50 \%$ & - & - & 4 \\
\hline Frecuencia respiratoria & & & \\
\hline 24-28 por minuto & 1 & - & - \\
\hline$>28$ por minuto & 4 & - & - \\
\hline $\begin{array}{l}\text { Puntaje total } \\
\text { Interpretación por } \\
\text { categorías de riesgo }\end{array}$ & $0-15$ & $0-19$ & $0-20$ \\
\hline Riesgo bajo (puntaje) & $0-2$ & $0-3$ & $0-4$ \\
\hline Riesgo promedio (IC 95\%) & $8 \%(7-9 \%)$ & $4 \%(3-4 \%)$ & $6 \%(5-6 \%)$ \\
\hline Riesgo moderado (puntaje) & $3-7$ & $4-9$ & $5-9$ \\
\hline Riesgo promedio (IC 95\%) & $32 \%(30-34 \%)$ & $30 \%(27-32 \%)$ & $32 \%(29-34 \%)$ \\
\hline Riesgo alto (puntaje) & $\geq 8$ & $\geq 10$ & $\geq 10$ \\
\hline Riesgo promedio (IC 95\%) & $73 \%(70-75 \%)$ & $80 \%(78-82 \%)$ & $80 \%(78-82 \%)$ \\
\hline
\end{tabular}

IMC = índice de masa corporal; TAC = tomografía computarizada; $I C=$ intervalo de confianza. ${ }^{*}$ Por encima de su límite de corte.

Modificado de: Mejía-Vilet JM, Córdova-Sánchez BM, Fernández-Camargo DA et al. Derivation of a score to predict admission to intensive care unit in patients with COVID-19: the ABC-GOALS Score. medRxiv. Available in: https://doi.org/10.1101/2020.05.12.20099416. ${ }^{15}$ sible transmisión por sangre podría ser una fuente de transmisión potencial en órganos de donantes fallecidos. En cambio, otros han reportado que en ninguna de las muestras por BAL fueron positivos y sólo $60 \%$ de aquellas por hisopado nasofaríngeo, concluyendo que el hisopado nasofaríngeo no fue suficiente para aislar el virus. ${ }^{23}$

El abordaje diagnóstico se realizó generalmente en etapas tempranas debido al antecedente de contacto con un caso confirmado, incluso se identificó que altas cargas virales tenían mayor capacidad de transmisión durante los primeros días de inicio de la sintomatología. Similar al reporte de Zou y su grupo, ${ }^{24}$ en el cual muestras obtenidas del tracto respiratorio superior en sujetos con síntomas tempranos tenían mayor carga viral, sobre todo en secreciones obtenidas por hisopado nasal más que en hisopado faríngeo. Incluso, en sujetos con completa resolución de síntomas, todavía se lograba una detección mínima del virus. Por lo anterior, la recomendación basada en obtener dos muestras negativas en pacientes asintomáticos para poder egresarlos, podría no generalizarse en todos los individuos, así que cada centro tendrá que establecer sus criterios de egreso y seguimiento ambulatorio.

\section{PRUEBAS SEROLÓGICAS}

Con respecto a las pruebas serológicas que identifican la presencia de anticuerpos (por ejemplo: IgG, IgM) de SARS-CoV-2, inicialmente la FDA estableció que su utilidad era limitada debido a que era necesario una evaluación exhaustiva, ya que un resultado negativo no excluiría la infección y requeriría un seguimiento con pruebas diagnósticas moleculares, incluso una prueba serológica positiva podía resultar de una infección previa o actual por otros coronavirus tales como HKU1, NL63, OC43 o 229E. ${ }^{25}$

Sin embargo, desde el mes de abril, existen en el mercado americano más de 70 pruebas serológicas (hasta ahora se ignora la proporción de falsos positivos y falsos negativos), así como la duración en la persistencia de anticuerpos lgG e lgM, incluso se desconoce si existe una respuesta humoral que confiera inmunidad ante una segunda infección. El significado clínico de la prueba serológica y molecular se puede interpretar acorde con la Tabla 4.

\section{DIAGNÓSTICO RADIOLÓGICO}

La tomografía computada (TAC) de tórax es un método de rutina para el diagnóstico de neumonía, ya que se 
caracteriza por ser sencillo, práctico y fácil de realizar. Los primeros reportes describieron en pacientes con COVID-19 imágenes típicas tales como opacidades en vidrio despulido, consolidación multifocal en parches y/o cambios intersticiales con distribución periférica. ${ }^{26}$

Teniendo como referencia RT-PCR, la TAC es una herramienta diagnóstica con alta sensibilidad, baja especificidad, moderado valor predictivo positivo y negativo que va alrededor de $97,25,65$ y $83 \%$, respectivamente. ${ }^{27}$ En casos con RT-PCR negativa, $70 \%$ tendrán una TAC sospechosa con imágenes típicas caracterizadas por un patrón de vidrio esmerilado. Se deben considerar algunos diagnósticos diferenciales por el tiempo de evolución ( $\leq 4$ semanas), como: otras infecciones virales como influenza, bacterias atípicas, edema pulmonar, reacción farmacológica, hemorragia pulmonar, daño agudo pulmonar por inhalación, neumonía eosinofílica aguda y neumonía intersticial aguda. También hay entidades crónicas ( $\geq 4$ semanas) que se deben descartar, tales como: enfermedad pulmonar intersticial, neumonía organizada, neumonía eosinofílica crónica, vasculitis, adenocarcinoma de pulmón, fibrosis focal o proteinosis alveolar pulmonar. Es así que considerando los diagnósticos previos y excluyendo los menos probables, podría definirse como caso altamente sospechoso en aquéllos con RT-PCR negativa pero con TAC positiva, después de un análisis exhaustivo de la sintomatología, hallazgos radiológicos y cambios en TAC posteriores. Por lo tanto, la TAC de tórax es una herramienta con alta sensibilidad y práctica para el diagnóstico y seguimiento de casos en zonas altamente endémicas.

\section{ESCALA PRONÓSTICA}

Se estima que entre 5-33\% de los pacientes con infección por SARS-CoV-2 requerirán manejo en una Unidad de Cuidados Intensivos (UCl). ${ }^{28,29}$ En México no todos los hospitales cuentan con áreas para el cuidado de pacientes críticos, es por ello que la implementación de una escala pronóstica puede ser una herramienta útil en la identificación de sujetos con características clínicas, de laboratorio e imagen que requieran ser derivados oportunamente a la $\mathrm{UCI}$. En un reporte reciente, Mejía-Vilet y sus colegas ${ }^{15}$ diseñaron un modelo predictivo de riesgo (ABC-GOALS, ver Tabla 5) en pacientes mexicanos que ingresaron a primer contacto al área de urgencias con el objetivo de identificar a aquéllos que requerirán atención en la $\mathrm{UCl}$, el desempeño de dichos modelos fue bueno, encontrando un área bajo la curva de $0.79,0.86$ y 0.88 para el mo- delo clínico, laboratorio y de imagen, respectivamente. El rendimiento para pronosticar mortalidad tuvo una sensibilidad de 31, 58 y 64\%, especificidad de 97,92 y $94 \%$ para el modelo clínico, laboratorio y de imagen, respectivamente. Esta herramienta requiere validación externa en otras poblaciones y ajustarse a otras regiones del país; sin embargo, su utilidad en nuestro medio resulta prometedora y sencilla de aplicar.

En conclusión, los receptores de trasplante renal pueden presentar un cuadro clínico similar a lo observado en la población general; no obstante, la severidad del cuadro será ligeramente mayor, por lo que será más frecuente que amerite manejo intrahospitalario, aunque son escasas las series reportadas hasta el momento. La inmunosupresión, sobre todo los inhibidores de calcineurina, pudieran participar en la supresión y regulación de la tormenta de citocinas. El diagnóstico molecular con RT-PCR se considera el estándar de oro; sin embargo, su sensibilidad está por debajo del $80 \%$. Sin duda, las circunstancias epidemiológicas, la sospecha clínica, RTPCR y los estudios de imagen son complementarios a la hora de establecer un correcto diagnóstico.

\section{REFERENCIAS}

1. López V, Vázquez $\mathrm{T}$, Alonso-Titos $\mathrm{J}$ et al. Recommendations on management of the SARS-CoV-2 coronavirus pandemic (Covid-19) in kidney transplant patients. Nefrologia. 2020. pii: S0211-6995(20)30037-0. doi: 10.1016/j.nefro.2020.03.002.

2. Guan $\mathrm{W}, \mathrm{Ni} Z, \mathrm{Hu}$ Y, Liang $\mathrm{W}, \mathrm{Ou} \mathrm{C}, \mathrm{He} J$ et al. Clinical characteristics of coronavirus disease 2019 in China. N Engl J Med. 2020; 382 (18): 1708-1720.

3. Zhou F, Yu T, Du R, Fan G, Liu Y, Liu Z et al. Clinical course and risk factors for mortality of adult inpatients with COVID-19 in Wuhan, China: a retrospective cohort study. Lancet [Internet]. 2020 [cited 2020 Mar 11].

4. Wang D, $\mathrm{Hu} \mathrm{B}, \mathrm{Hu} \mathrm{C}$ et al. Clinical characteristics of 138 hospitalized patients with 2019 novel coronavirus-infected pneumonia in Wuhan, China. JAMA. 2020. doi: 10.1001/ jama.2020.1585.

5. Bello-Chavolla O, Bahena-López J, Antonio-Villa E et al. Predicting mortality due to SARS-CoV-2: A mechanistic score relating obesity and diabetes to COVID-19 outcomes in Mexico. medRxiv. 2020 [preprint]. Available in: https://doi. org/10.1101/2020.04.20.20072223

6. Marx D, Moulin B, Fafi-Kremer S et al. First case of COVID-19 in a kidney transplant recipient treated with belatacept. Am J Transplant. 2020. doi: 10.1111/ajt.15919.

7. Boletín Informativo Especial Centro Nacional de Trasplantes (CENATRA): Casos reportados con COVID-19 en personas receptoras de trasplante [última actualización 25 de mayo del 2020]. [Acceso 28 de mayo del 2020] Disponible en: https:// www.gob.mx/cenatra/es/documentos/boletin-informativoespecial-trasplantes-y-covid-19?state=published

8. FAQs on Diagnostic Testing for SARS-CoV-2. Available in: https://www.fda.gov/medical-devices/emergency-situationsmedical-devices/faqs-diagnostic-testing-sars-cov-2. 
9. Wang $\mathrm{W}, \mathrm{Xu} \mathrm{Y}, \mathrm{Gao} \mathrm{R}$ et al. Detection of SARS-CoV-2 in different types of clinical specimens. JAMA. 2020; 323 (18): 1843-1844. doi: 10.1001/jama.2020.3786.

10. Wu Z, McGoogan JM. Characteristics of and important lessons from the coronavirus disease 2019 (COVID-19) outbreak in china: summary of a report of 72314 cases from the Chinese Center for Disease Control and Prevention. JAMA. 2020. doi: 10.1001/jama.2020.2648.

11. Lechien JR, Chiesa-Estomba CM, Place $S$ et al. Clinical and epidemiological characteristics of 1,420 european patients with mild-to-moderate coronavirus disease 2019. J Intern Med. 2020. doi: 10.1111/joim.13089.

12. Grasselli G, Zangrillo A, Zanella A et al. Baseline Characteristics and outcomes of 1591 patients infected with SARS-CoV-2 admitted to ICUs of the Lombardy region, Italy. JAMA. 2020. doi: 10.1001/jama.2020.5394.

13. Bialek S, Boundy E, Bowen V, et al. Severe outcomes among patients with coronavirus disease 2019 (COVID-19) - United States, February 12-march 16, 2020. Morb Mortal Wkly Rep. 2020; 69 (12): 343-346. doi:10.15585/mmwr.mm6912e2

14. Arentz M, Yim E, Klaff $L$ et al. Characteristics and outcomes of 21 critically ill patients with COVID-19 in Washington State. JAMA. 2020. doi: 10.1001/jama.2020.4326.

15. Mejía-Vilet JM, Córdova-Sánchez BM, Fernández-Camargo DA et al. Derivation of a score to predict admission to intensive care unit in patients with COVID-19: the ABC-GOALS Score. medRxiv. 2020. Available in: https://doi.org/10.1101/2020.05.12.20099416

16. Zhu L, Xu X, Ma K et al. Successful recovery of COVID-19 pneumonia in a renal transplant recipient with long-term immunosuppression. Am J Transplant. 2020. doi: 10.1111/ ajt.15869.

17. Qiuyu Li, Qin Cheng, Zhiling Zhao, Lin Zeng, Lan Zhu, Wei Guo, Chao Li , Junhong Wang, Shu Li, Ning Shen QG. Novel Coronavirus Infection and Acute Kidney Injury in Two Renal Transplant Recipients: Case Report. preprints.org. Published online 2020. https://www.preprints.org/manuscript/202003.0190/v1

18. Li F, Cai J, Dong N. First cases of COVID-19 in heart transplantation from China. J Hear Lung Transplant. 2020; 39 (5): 496-497. doi:10.1016/j.healun.2020.03.006

19. Qin J, Wang $H$, Qin X et al. Perioperative Presentation of COVID-19 Disease in a Liver Transplant Recipient. Hepatology. 2020. doi: 10.1002/hep.31257.
20. Banerjee D, Popoola J, Shah S, Ster IC, Quan V, Phanish M. COVID-19 infection in kidney transplant recipients. Kidney Int. 2020; 97 (6): 1076-1082. doi: 10.1016/j.kint.2020.03.018.

21. Pereira MR, Mohan S, Cohen DJ et al. COVID-19 in solid organ transplant recipients: initial report from the US Epicenter. Am J Transplant. 2020. doi: 10.1111/ajt.15941.

22. Akalin E, Azzi Y, Bartash $\mathrm{R}$ et al. Covid-19 and Kidney Transplantation. N Engl J Med. 2020. doi: 10.1056/ NEJMc2011117.

23. Lescure FX, Bouadma L, Nguyen D et al. Clinical and virological data of the first cases of COVID-19 in Europe: a case series. Lancet Infect Dis. 2020; 20 (6): 697-706. doi: 10.1016/S1473-3099(20)30200-0.

24. Zou L, Ruan F, Huang M et al. SARS-CoV-2 viral load in upper respiratory specimens of infected patients. N Engl J Med. 2020; 382 (12): 1177-1179. doi: 10.1056/NEJMc2001737.

25. Policy for Diagnostic Tests for Coronavirus Disease-2019 during the Public Health Emergency Immediately in Effect Guidance for Clinical Laboratories, Commercial Manufacturers, and Food and Drug Administration Staff. [29/03/2020] Available in: https:// www.fda.gov/media/135659/download

26. Chung M, Bernheim A, Mei X et al. CT imaging features of 2019 novel coronavirus (2019-nCoV). Radiology. 2020. doi: 10.1148/ radiol.2020200230.

27. Ai T, Yang Z, Hou H et al. Correlation of chest CT and RT-PCR testing in coronavirus disease 2019 (COVID-19) in China: a report of 1014 cases. Radiology. 2020; 200642. doi: 10.1148/ radiol.2020200642.

28. Goyal P, Choi JJ, Pinheiro LC et al. Clinical characteristics of Covid-19 in New York City. N Engl J Med. 2020. doi: 10.1056/ NEJMc2010419.

29. Richardson S, Hirsch JS, Narasimhan M et al. Presenting characteristics, comorbidities, and outcomes among 5700 patients hospitalized with COVID-19 in the New York City Area. JAMA. 2020. doi: 10.1001/jama.2020.6775.

Correspondencia:

Dra. Idalia Parra-Ávila

Departamento de Trasplantes.

Unidad Médica de Alta Especialidad,

IMSS Mérida.

E-mail: dra.idaliaparra@gmail.com 